\title{
Visual threshold as a function of test area and caffeine administration*
}

\author{
A. LEONARD DIAMOND \\ University of Hawaii, Honolulu, Hawaii 96822 \\ and \\ ROBERT E. COLE \\ Simon Fraser University, Burnaby, B.C., Canada
}

The threshold luminance of a small circle was studied as a function of its area (varied from $2.69 \mathrm{~min}$ to $26.87 \mathrm{~min}$ in radius) and for different dosages of caffeine $(0,1.5$, and 3 grains). The zero caffeine condition shows the classical decrease in threshold with increased area. With caffeine administration, the slope of this threshold-area function remains unchanged, although the entire function shifts downward (threshold decreases equally for all areas) increasingly with an increasing amount of caffeine. The results combined with previous caffeine experiments suggest a theoretical interpretation that caffeine causes the "on" visual pathways to become more sensitive to light.

The threshold luminance necessary to see a small single visual field decreases as the area of the field increases (Graham, Brown, \& Mote, 1939; Diamond, 1962). This is an illustration of single-field brightness enhancement as suggested by Diamond (1960). That is, the sensitivity to light is enhanced as the area of a field is increased.

Heinemann (1955) found the brightness of a test field of constant luminance increased (was enhanced) when he surrounded the test field with an annulus of very low luminance. This is an illustration of multiple-field enhancement. Kleman et al (1961) repeated Heinemann's results and further found that administration of three grains of caffeine to the $S s$ resulted in the apparent disappearance of the enhancement.

It is this elimination of enhancement by caffeine that is the interest of the present investigation. The question is, will caffeine eliminate or reduce enhancement in a single field as well as in the Kleman multiple-field visual situation?

The present experiment, designed to test this question, measures the threshold luminance of a test field as its area is increased from $2.69 \mathrm{~min}$ to $26.87 \mathrm{~min}$ in radius. This is done for three dosages of caffeine, 0, 1.5, and 3.0 grains. (Kleman used 3 grains.) It is expected that threshold luminance will decrease as a function of test area, illustrating enhancement. On the other hand, if caffeine is effective in reducing enhancement, the slope of the threshold decrease should lessen with increased caffeine.

*The research reported here was supported in part by Grant GB-5768 from the National Science Foundation and Grant NB-06890 from the Institute of Neurological Diseases and Blindness, USPHS.

\section{APPARATUS}

The apparatus, presented in Fig. 1, consists of a light path to S's right eye. A 150-W frosted tungsten-filament bulb operated at $115 \mathrm{~V}$ ac constitutes the light source. The light, diffused by flashed opal glass, passes through 1 of 10 test circles (of different areas), a crossed Polaroid filter (for continuous luminance control), and fixed-density Wratten filters (for discrete luminance control). Next, the light is made parallel by a 4-diopter lens, and finally it passes through a $3-\mathrm{mm}$ artificial pupil to the eye of $S$, who is seated in a dark room. The artificial pupil is mounted in an optician's trial frame fixed to S's head in order to reduce variability of brightness judgments due to head movements (Diamond, 1955).

A fixation point, a 3 -min point of light, is located 43 min nasally from the center of the test field. Its source is a $50-\mathrm{W}$ tungsten filament projection bulb operated at $115 \mathrm{~V}$ ac. Its point of introduction to the threshold light path between the variable and fixed Polaroid (see Fig. 1) is designed to permit constant fixation point luminance with variation of the variable Polaroid and, therefore, test luminance. The $\mathrm{S}$ is instructed to fixate this $3-\mathrm{min}$ point of light and report the presence or absence of the test field as $E$ manipulates the variable Polaroid. Since the visual angle between the fixation point and the outer edge of the test field does not exceed $70 \mathrm{~min}$, retinal stimulation can be considered essentially foveal.

Test-field areas are varied in the following manner: 10 circular patterns, increasing in diameter in 0.0156 -in. steps from 0.0156 in. to $0.1560 \mathrm{in}$., are cut in a thin metal disk that can be rotated and set so that any one pattern will fall directly in front of the diffuse light source in the optical path.

In addition to the apparatus elements shown in Fig. 1, the optical pathway also includes a Dove prism and a circular prism. However, these elements are nonfunctional in the present study.

\section{PROCEDURE}

Threshold luminance was explored as a function of the 10 test-field areas, their radii ranging from $2.69 \mathrm{~min}$ to $26.87 \mathrm{~min}$ in visual angle, under the following three experimental conditions: (1) The first consisted of oral administration of a placebo capsule containing 5 grains of calcium lactate; (2) the second consisted of administration of a capsule containing 1.5 grains of caffeine; and (3) the third consisted of administration of a capsule containing 3.0 grains of caffeine.

Experimental sessions were the same for each $S$ for each experimental day. Fifteen minutes before entering the darkroom, $S$ was given the placebo. At the end of this period, $\mathrm{S}$ entered the darkroom, adjusted the optician's trial frame, and then dark adapted for $15 \mathrm{~min}$. Next, the S's range of thresholds was determined through the E's concurrent manipulation of the Wratten filters and variable Polaroid, and the $S$ reported on the presence or absence of the test field while focusing on the fixation point. Once the range of thresholds for a particular session was established, all subsequent luminance variations could be accomplished with a variable Polaroid alone. Threshold measurements were then

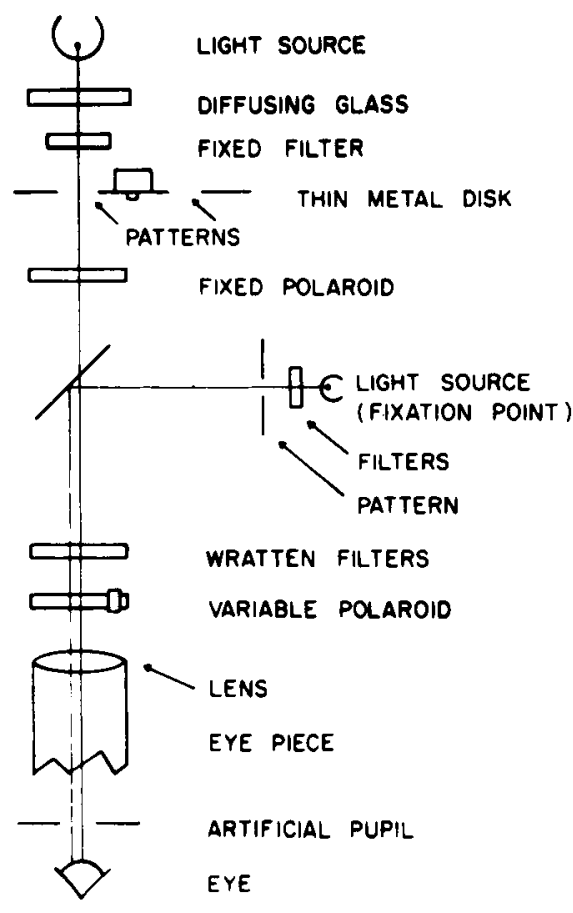

Fig. 1. Apparatus. 
Table 1

Threshold Luminance as a Function of Test Radius for Caffeine and Placebo Conditions (in Log mL). (Data are for Ss JN. SI, and TH.) Drug Condition

\begin{tabular}{|c|c|c|c|c|c|c|c|c|c|c|c|c|c|c|c|c|}
\hline \multirow{3}{*}{$\begin{array}{l}\text { Test Radius } \\
\text { (Minutes) }\end{array}$} & \multicolumn{8}{|c|}{1.5 Grains } & \multicolumn{8}{|c|}{3.0 Grains } \\
\hline & \multicolumn{4}{|c|}{ Placebo } & \multicolumn{4}{|c|}{ Caffeine } & \multicolumn{4}{|c|}{ Placebo } & \multicolumn{4}{|c|}{ Caffeine } \\
\hline & $\mathrm{JN}$ & SI & TH & Avg. & $\mathrm{JN}$ & SI & $\mathrm{TH}$ & Arg. & $3 \mathrm{X}$ & SI & $\mathrm{TH}$ & Avg. & $\mathbf{J N}$ & SI & $\mathrm{TH}$ & Avg. \\
\hline $\begin{array}{r}2.69 \\
5.38 \\
8.07 \\
10.76 \\
13.45 \\
16.14 \\
18.83 \\
21.52 \\
24.21 \\
26.87 \\
\end{array}$ & $\begin{array}{l}-2.27 \\
-2.37 \\
-2.47 \\
-2.46 \\
-2.60 \\
-2.64 \\
-2.74 \\
-2.80 \\
-2.90 \\
-2.89 \\
\end{array}$ & $\begin{array}{l}-1.86 \\
-1.92 \\
-2.06 \\
-2.15 \\
-2.24 \\
-2.32 \\
-2.46 \\
-2.50 \\
-2.64 \\
-2.61 \\
\end{array}$ & $\begin{array}{l}-2.00 \\
-2.10 \\
-2.21 \\
-2.31 \\
-2.48 \\
-2.54 \\
-2.55 \\
-2.66 \\
-2.74 \\
-2.77 \\
\end{array}$ & $\begin{array}{l}-2.04 \\
-2.13 \\
-2.25 \\
-2.31 \\
-2.44 \\
-2.50 \\
-2.58 \\
-2.65 \\
-2.76 \\
-2.76 \\
\end{array}$ & $\begin{array}{l}-2.26 \\
-2.34 \\
-2.43 \\
-2.56 \\
-2.61 \\
-2.71 \\
-2.74 \\
-2.79 \\
-2.82 \\
-2.92 \\
\end{array}$ & $\begin{array}{l}-1.90 \\
-2.02 \\
-2.26 \\
-2.26 \\
-2.32 \\
-2.40 \\
-2.49 \\
-2.51 \\
-2.65 \\
-2.72 \\
\end{array}$ & $\begin{array}{l}-2.22 \\
-2.30 \\
-2.40 \\
-2.50 \\
-2.58 \\
-2.68 \\
-2.73 \\
-2.84 \\
-2.88 \\
-2.86 \\
\end{array}$ & $\begin{array}{l}-2.13 \\
-2.22 \\
-2.36 \\
-2.44 \\
-2.50 \\
-2.60 \\
-2.65 \\
-2.71 \\
-2.78 \\
-2.83 \\
\end{array}$ & $\begin{array}{r}-2.36 \\
-2.53 \\
-2.59 \\
-2.63 \\
-2.74 \\
-2.85 \\
-2.92 \\
-3.06 \\
-3.20 \\
-3.26\end{array}$ & $\begin{array}{l}-1.13 \\
-1.32 \\
-1.47 \\
-1.54 \\
-1.62 \\
-1.75 \\
-1.87 \\
-1.93 \\
-1.98 \\
-2.00\end{array}$ & $\begin{array}{l}-2.23 \\
-2.39 \\
-2.57 \\
-2.58 \\
-2.79 \\
-2.91 \\
-2.98 \\
-3.00 \\
-3.12 \\
-3.13 \\
\end{array}$ & $\begin{array}{l}3-1.91 \\
-2.08 \\
-2.21 \\
-2.28 \\
-2.38 \\
-2.50 \\
-2.59 \\
-2.66 \\
-2.77 \\
-2.80 \\
\end{array}$ & $\begin{array}{l}-2.72 \\
-2.78 \\
-2.83 \\
-2.90 \\
-3.05 \\
-3.16 \\
-3.22 \\
-3.26 \\
-3.40 \\
-3.44 \\
\end{array}$ & $\begin{array}{l}-1.26 \\
-1.37 \\
-1.57 \\
-1.64 \\
-1.80 \\
-1.93 \\
-2.01 \\
-2.09 \\
-2.11 \\
-2.18 \\
\end{array}$ & $\begin{array}{l}-2.33 \\
-2.47 \\
-2.57 \\
-2.72 \\
-2.82 \\
-2.94 \\
-2.96 \\
-3.06 \\
-3.14 \\
-3.14 \\
\end{array}$ & $\begin{array}{l}-2.10 \\
-2.21 \\
-2.32 \\
-2.42 \\
-2.56 \\
-2.68 \\
-2.73 \\
-2.80 \\
-2.88 \\
-2.92 \\
\end{array}$ \\
\hline
\end{tabular}

taken for the 10 test-field areas, the order being from smallest to largest.

At the conclusion of the placebo condition, $S$ left the darkroom for a $1-h$ rest. Thirty minutes before reentering the darkroom, $\mathrm{S}$ was given either a 1.5 -grain or a 3.0-grain capsule of caffeine, depending on the caffeine level to be tested during that session. The procedure described above under the placebo condition was then repeated. The order of presentation of the 1.5-grain condition and the 3.0-grain condition was randomized.

Two females, JN and SI, and one male, $\mathrm{TH}$, served as Ss. All Ss were fully trained in the adjustment of the optician's trial frames and in focusing on the fixation point while making judgments. In an exploratory study, $\mathrm{JN}$ and $\mathrm{TH}$ were tested for placebo effects with the results showing no significant differences between threshold judgments under placebo vs nonplacebo conditions. Although the possibility of counterbalancing the three conditions was considered, it was decided not practicable, since caffeine has been found to affect visual behavior for as long as $6 \mathrm{~h}$ following administration (Kleman, Diamond, \& Smith, 1961).

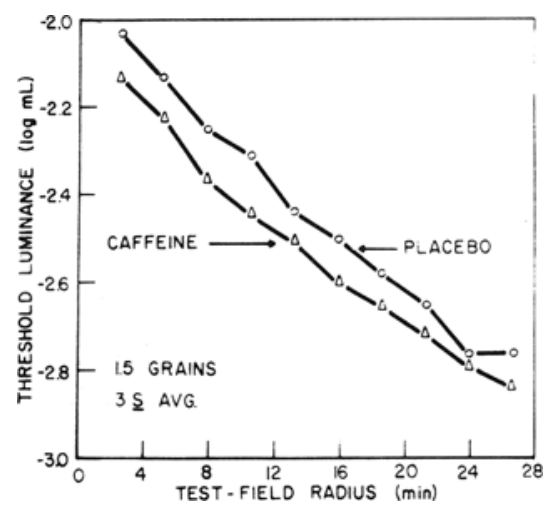

Fig. 2. Threshold in $\log m L$ as a function of test-field radius for $1 \frac{1 / 2}{2}$ grains of caffeine vs no caffeine (placebo condition).
During the above procedure, the psychophysical method of limits was used as described by Stevens (1951). From his position outside the darkroom. E slowly and evenly varied the luminance of the test field by moving the variable Polaroid filter in the optical pathway in a counterbalanced order of ascending and descending series. As the $E$ slowly increased the luminance of the test field, $S$ was instructed to inform the $E$ when the entire test field with its boundaries appeared. Conversely, as the E slowly decreased the luminance of the test field, the $S$ was instructed to inform the $E$ when the boundaries of the test field became indistinct. The mean of three such pairs of ascending-descending judgments was taken as a measure of the test-field threshold luminance.

\section{RESULTS}

The data are presented in Table 1. The means of threshold luminances ( $\log \mathrm{mL}$ ) are given separately for the three $S$ s for different days and for 10 test-field areas in addition to the combined means of the Ss. The latter values are graphed in Figs. 2 and 3.

The placebo condition curves show the enhancement phenomenon found by Graham, Brown, \& Mote (1939) and Diamond (1962); i.e., as test-field area increases, test-field threshold decreases. With caffeine administration, furthermore, there is a decrease (from the placebo values) in the entire threshold function. This overall decrease, moreover, grows more pronounced as caffeine dosage is increased from 1.5 to 3.0 grains. There seems to be no systematic change, however, in the slope of the threshold-area curve as caffeine dosage is increased from 0 to 3 grains.

DISCUSSION
Since the results show no systematic change in the slope of the threshold-area curve with caffeine dosage increase. it seems reasonable to conclude that caffeine does not affect single-field enhancement, at least for the dosage ( 3 grains) that affects multiple-field enhancement.

Threshold does decrease systematically. however, over the entire threshold-area curve with increased caffeine dosage. The decrease itself is consistent with the results of Ditchburn \& Power Steele (1941). This result raises an interesting theoretical possibility with respect to underlying physiological events.

Kleman et al (1961), as mentioned above. found that enhancement decreases with caffeine administration in the multiple-field situation (a test circle surrounded by an annulus). One speculation is that "on" fibers in the retina (see Hartline, 1940) become more sensitive with caffeine administration and, as a result, depression of the test field by the increasingly active inducing-field "on" fibers increases, and thus reduces or cancels out enhancement. Unfortunately, since his measure was a binocular match, Kleman's data does not allow detection of changes in the absolute sensitivity of the eyes, since both eyes of the Ss presumably were affected equally by the caffeine.

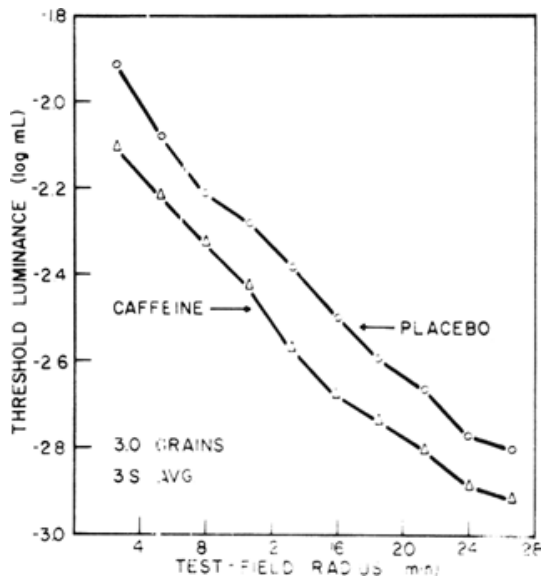

Fig. 3. Threshold in $\log \mathrm{mL}$ as a function of test-field radius for three grains of caffeine vs no caffeine (placebo condition). 
On the other hand, the threshold measurement in the present experiment does indicate absolute sensitivity. And since the data show that absolute sensitivity increases (threshold energy decreases) with caffeine administration, this fact lends support to the speculation that retinal "on" fibers become more sensitive with caffeine.

Why then was enhancement also not affected in the present threshold experiment, as it was in Kleman's brightness match experiment? One explanation may be in the probable constancy of "on" fiber activity for a threshold response. That is, regardless of the amount of stimulus or whether or not caffeine has been administered, we might assume that the minimal amount of physiological activity necessary to produce the threshold response remains constant, since the threshold sensation by definition and by instruction is presumed constant. For threshold measurement in the present experiment, therefore, although the "on" fibers might become more sensitive, the amount of "on" activity remains the same regardless of the administration of caffeine. If "on" activity, therefore, is in fact constant, we would predict no change in enhancement. (In Kleman's experiment, "on" activity might be assumed to have increased with caffeine administration although, as pointed out above, his binocular match procedure did not provide a measure of such a change.)

One further consideration involves the role of the "off" fiber in the retina. Diamond (1960) suggests that both singleand multiple-field enhancement can be explained in terms of an inhibition of "off" retinal activity resulting in a disinhibition (see Hartline \& Ratliff, 1959) of test brightness. In the single-field situation, as the test area increases, its light turns off more and more "off" fibers that were initially depressing the test-field's "on" activity (see Granit, 1955, for a discussion of "on-off" antagonism). For Kleman's multiple-field enhancement, the introduction of a low luminance surround theoretically eliminates "off" activity in the area surrounding the test field and again the disinhibited test brightness increases

Since enhancement did not change in the present experiment, then, according to the above theoretical account, "off" fiber activity probably was not affected by caffeine administration, even though "on" fiber sensitivity was. In this connection, it is interesting to note that a number of investigators suggest differences between "off" and "on" retinal systems (MacNichols \& Love, 1960; Ratliff, 1965), whereas others suggest that the "off" response might be a "rebound" (Florey, 1961) or a "net summation" (Kennedy, 1963) of the "on" responses. The results of the present study, interpreted in the light of Diamond's theoretical account (1960), would tend to support the idea of some functional difference between "on" and "off" systems and that this difference, whatever it might be, shows up as a differential response to caffeine, the "on" system being made more sensitive to stimuli and the "off" system not significantly affected.

\section{REFERENCES}

DIAMOND, A. L. Foveal simultaneous contrast as a function of inducing-field area. Journal of Experimental Psychology, 1955, 50, 14-152.

DIAMOND, A. L. A theory of depression and enhancement in the brightness response. Psychological Review, 1960, 67, 168-199.

DIAMOND, A. L. Brightness of a field as a function of its area. Journal of the Optical Society of America, 1962, 52, 700-706.

DITCHBURN, R. W., \& POWER-STEELE, E. J. Effect of caffeine and bromide on dark adaptation. Nature, London, 1941, 1, 745-746.

FLOREY, E. Nervous inhibition: Proceedings of the Second Friday Harbor Symposium. New York: Pargamon Press, 1961. Pp. 318-325.
GRAHAM, C. H., BROWN, R. H., \& MOTE, F. $A$. The relation of size of stimulus and intensity in the human eye: I. Intensity thresholds for white light. Journal of Experimental Psychology, 1939, 24, 555-573.

GRANIT, R. Receptors and sensory perception. New Haven: Yale University Press, 1955.

HARTLINE, H. K. The effects of spatial summation in the retina of the excitation of the fibers of the optic nerve. American Journal of Physiology, 1940, 130, 700-711.

HARTLINE, H. K., \& RATLIFF, F. Inhibitory interaction of receptor units in the eye of Limulus. Journal of General Physiology, 1957, 40, 357-376.

HEINEMANN, E. G. Simultaneous brightness induction as a function of inducing and test-field luminances. Journal of Experimental Psychology, 1955, 50, 89-96.

KENNEDY, D. Inhibition in visual systems. Scientific American, July 1963, 2-8.

KLEMAN, J. P., DIAMOND, A. L., \& SMITH, E. Effects of caffeine on enhancement in foveal simultaneous contrast. Journal of Experimental Psychology, 1961, 61, 18-2.

MacNICHOL, E. F., JR., \& LOVE, W. E. Electrical responses of the retinal nerve and optic ganglia of the spine. Science, 1960, 132, 737-738.

RATLIFF, F. Mach bands: Quantitative studies on neural networks in the retina. San Francisco: Holden-Day, 1965.

STEVENS, S. S. Handbook of experimental psychology. New York: Wiley, 1951.

\title{
Short-term retention and similarity of word length
}

\author{
HAROLD L. HAWKINS and R. HAL SHIGLEY \\ University of South Florida, Tampa, Fla. 33620
}

The short-term retention of lists containing word quadruples identical in length was measured by the Peterson method. Nine blocks of three lists were presented. The successively presented lists comprising a block contained words identical in length, 3,6 , or 9 letters, while word length varied across blocks. Overall retention declined with increasing word length. Retention also declined with increasing number of successive lists containing words of a common length and increased when immediately preceding lists contained words of different length, indicating that proactive interference varies as a function of the similarity in length of recently stored items.

Keppel \& Underwood (1962) have demonstrated that a major portion of the forgetting observed in short-term retention under conditions first described by Peterson \& Peterson (1959) is attributable to proactive interference (PI). Subsequently, several investigators have shown that PI can be effectively manipulated in the Peterson situation by varying the similarity relationship between adjacent lists (Wickens, Born, \& Allen, 1963; Loess, 1968; Hawkins, Martini, \& Sayre, 1969). In the Wickens et al study, Ss were initially tested on a series of lists consisting exclusively of either consonant or digit trigrams. A second series contained trigrams of either the same class or of the alternate class. When trigram class remained constant across series, marked PI was observed in as few as three trials. However, when trigram class altered, PI was not evident, indicating a "release from PI" with changing material. Comparable results were reported by Loess (1968) in a study varying the taxonomic category of successive word triads and by Hawkins et al (1969) in a study varying interlist acoustic similarity. 